\title{
State aggregation-based model of asynchronous multi-fiber optical switching with shared wavelength converters
}

\author{
Nail Akar ${ }^{\mathrm{a}, *, 1}$, Carla Raffaelli ${ }^{\mathrm{b}}$, Michele Savi ${ }^{\mathrm{c}}$ \\ a Electrical and Electronics Engineering Department, Bilkent University, Ankara, Turkey \\ ${ }^{\mathrm{b}}$ DEI - University of Bologna, Bologna, Italy \\ ${ }^{\mathrm{c}}$ Department of Telematics - NTNU, Trondheim, Norway
}

\section{A R T I C L E I N F O}

\section{Article history:}

Received 14 May 2012

Accepted 7 December 2012

Available online 3 January 2013

\section{Keywords:}

Optical packet switching

Wavelength converters

Markov chains

State aggregation

\begin{abstract}
A B S T R A C T
This paper proposes new analytical models to study optical packet switching architectures with multi-fiber interfaces and shared wavelength converters. The multi-fiber extension of the recently proposed Shared-Per-Input-Wavelength (SPIW) scheme is compared against the multi-fiber Shared-Per-Node (SPN) scheme in terms of cost and performance for asynchronous traffic. In addition to using Markov chains and fixed-point iterations for modeling the mono-fiber case, a novel state aggregation technique is proposed to evaluate the packet loss in asynchronous multi-fiber scenario. The accuracy of the performance models is validated by comparison with simulations in a wide variety of scenarios with both balanced and imbalanced input traffic. The proposed analytical models are shown to remarkably capture the actual system behavior in all scenarios we tested. The adoption of multi-fiber interfaces is shown to achieve remarkable savings in the number of wavelength converters employed and their range. In addition, the SPIW solution allows to save, in particular conditions, a significant number of optical gates compared to the SPN solution. Indeed, SPIW allows, if properly dimensioned, potential complexity and cost reduction compared to SPN, while providing similar performance.
\end{abstract}

(c) 2013 Elsevier B.V. All rights reserved.

\section{Introduction}

In recent years, optical switching technology has entered a mature phase to support the ever growing bandwidth demands of user applications [1]. At the same time, emerging and future Internet-based services [2,3] to support these user applications call for enhanced flexibility and reconfigurability in transport networks. Packet-based optical networking based on either optical packet switching or optical burst switching, is the most suitable solutions to achieve high network reconfiguration capability and flexibility and has been widely studied and demonstrated as feasible in the last decade $[2,4]$.

\footnotetext{
* Corresponding author. Tel.: +90 312 2902337; fax: +90 3122664192. E-mail address: akar@ee.bilkent.edu.tr (N. Akar).

${ }^{1}$ The work of N. Akar is supported in part by TUBITAK project No. EEE111E106
}

One of the main drawbacks of packet-based optical networking is represented by contention which arises as a consequence of the need for resource sharing for optical packets within the network nodes. In conventional electronically switched networks, packet contention is solved in time domain by queuing packets and allowing resource sharing on a time division multiplexing basis. Unfortunately, queuing is not straightforward in optical switching with current optical technologies and contention is instead typically addressed by exploiting wavelength and space domains. Wavelength Converters (WCs) are employed in optical packet/burst switching to exploit the wavelength domain with the purpose of contention resolution. As a matter of fact, when two or more optical packets simultaneously need the same forwarding resource (optical gate, fiber interface, splitter/combiner, etc.) within a node, different wavelengths are used to encode them, by wavelength converting some of the optical signals, thus avoiding wavelength contention [5]. 
However, in spite of the progress in optical fabrication technology, all-optical WCs are still considered complex and expensive components [1]. For this reason, it is important to limit the number of WCs employed and try to exploit as simple WCs as possible in terms of implementation. In particular, WCs differ on the basis of their tunability and wavelength conversion range. In general, it can be assessed that fixed WCs are simpler to be fabricated with respect to tunable ones [6]. Furthermore, it has been demonstrated that the quantity and type of WCs employed to obtain a given packet loss performance are related to the specific switch architecture [7].

In order to reduce the number of WCs employed, different schemes for sharing WCs inside an optical switching node have been proposed in the past [8]. In particular, the Shared-Per-Node (SPN) sharing scheme provides the best packet loss performance since WCs are shared among all the incoming packets $[8,9]$. However, SPN requires tunable-input/tunable-output WCs, being the most complex type of WC, and also a relatively large number of optical gates to connect them. To simplify the complexity of the SPN scheme, the Shared-Per-InputWavelength (SPIW) optical switching architecture has been introduced which employs fixed-input/tunable-output WCs [10]. In this architecture, for each wavelength, there is a separate pool of WCs that can be used by all optical packets arriving on this particular wavelength. The SPIW architecture has been demonstrated to have superior properties in regards with its feasibility (fewer optical gates required) [11], power consumption [12] and complexity [9], while performing quite close to the sharedper-node architecture [7,9], as demonstrated in recent research studies $[13,14]$.

The conversion range required by each WC is related to the number of wavelength channels supported by each fiber interface. The adoption of multi-fiber interfaces allows to repeat the same wavelength as many times as the number of fibers allocated at each interface, being them spatially separated [15]. This solution was studied in the past in WDM circuit-switched networks to optimize resources in transport networks $[16,17]$. Recently, multi-fiber solutions based on the SPN and SPIW sharing scheme have been presented [9] in synchronous setting. Even though the synchronous operation mode guarantees better packet loss performance within a node than the asynchronous one, it requires expensive and complex synchronizers at the input channels. Moreover, synchronous operation requires in general a more complex management at network level. Hence, it is crucial to analyze the performance of the SPN and SPIW schemes in the simpler asynchronous operation. To the best of our knowledge, the study of the multi-fiber SPIW has not yet been performed in asynchronous context in the existing literature. Only the multi-fiber SPN solution has been studied with asynchronous operation [18], so the present paper proposes an analysis of the multi-fiber SPIW and a comparison between the two. Multi-fiber SPIW architecture seems to be attractive in asynchronous operation since it has promising properties in terms of feasibility and power consumption, together with the possibility to limit the conversion range [19]. In the remainder of this paper, the two architectures considered will be referred to as A-MF-SPN (Asynchronous Multi-Fiber Shared-PerNode) and A-MF-SPIW (Asynchronous Multi-Fiber Shared-Per-Input-Wavelength).

Based on previous motivations, the A-MF-SPIW switch architecture which shares fixed-input WCs is investigated in this paper to analytically obtain the packet loss performance. A similar kind of comparison was presented in [7] for the mono-fiber case whereas the current paper concentrates on the impact of multi-fiber switch interfaces. The assumptions behind the analytical model are:

- Optical packet arrivals to the switch are Poisson.

- Packet lengths are exponentially distributed.

- Packet traffic is allowed to be imbalanced across destination interfaces.

- Packet traffic is balanced across incoming wavelengths.

This paper is an extension of a recently published work in [14] where the analytical model for packet loss evaluation of the A-MF-SPIW scheme has been briefly presented. Compared with the work in [14], in this study:

- A complete description of a novel state aggregation technique to cope with multi-fiber interfaces is presented. Although various state aggregation schemes are available for studying large Markov chains, a stateaggregation method, specifically applied to the problem of interest, is proposed.

- The model is extended to cover the imbalanced input traffic case and to the analysis of the A-MF-SPN scheme as well.

- Validation section is provided where both A-MF-SPIW and A-MF-SPN models are compared with simulation, highlighting the accuracy achieved.

- Relying on the analytical models, the two architectures are extensively compared in terms of both packet loss performance and complexity, highlighting how the A-MF-SPIW not only exploits fixed-input WCs but also requires fewer optical gates in different configurations.

- Again, using the analytical model only, the achievable throughput for both architectures as a function of the number of fibers per interface is evaluated.

The paper is organized as follows. Section 2 describes the A-MF-SPN and A-MF-SPIW architectures and introduces related formulas for complexity evaluation. Section 3 presents the state aggregation-based methodology to calculate the packet loss probability in A-MF-SPN and A-MF-SPIW. Section 4 discusses model validation, performance comparison and complexity evaluation with respect to the main switch parameters. Finally, Section 5 provides the conclusions of this work.

\section{A-MF-SPN and A-MF-SPIW architectures}

This section provides the description of the two multifiber architectures considered in this paper. Section 2.1 introduces the A-MF-SPN architecture and Section 2.2 describes the A-MF-SPIW architecture. 


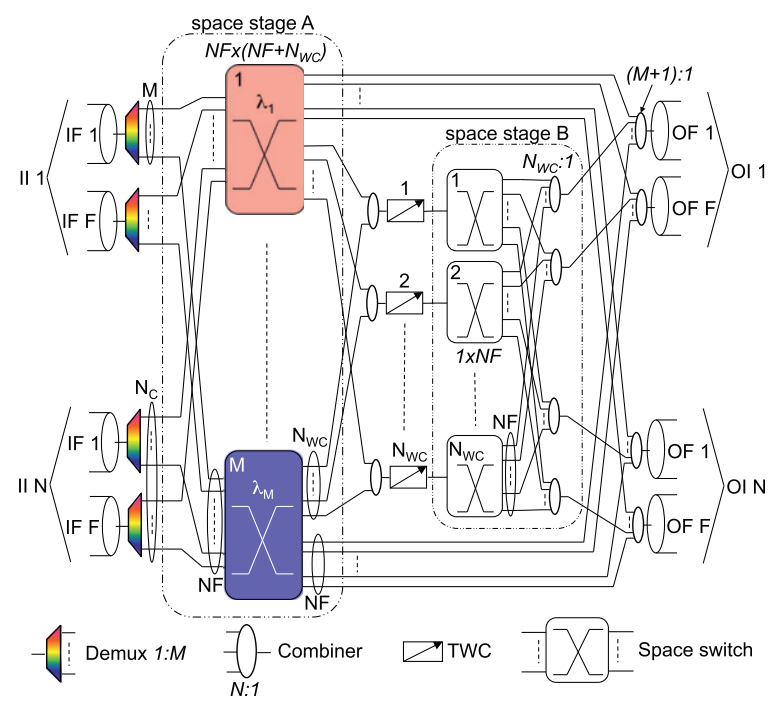

Fig. 1. A-MF-SPN architecture with $N$ input/output interfaces equipped with $F$ optical fibers carrying $M$ wavelengths each. The switch is equipped with $N_{W C}<N M F$ WCs shared per node.

\subsection{A-MF-SPN architecture}

The A-MF-SPN architecture (Fig. 1) consists of $N$ input/ output interfaces (II/OIs) each provided with $F$ optical fibers carrying $M$ wavelengths. Hence, the number of channels per interface is $N_{C}=M F$. This architecture addresses contention in the wavelength domain and for this purpose it is equipped with a pool of $N_{W C}(<N M F)$ WCs shared among all the input channels, so among all the incoming packets. For this reason, the $N_{W C}$ WCs must be tunable-input/tunable-output, since the incoming wavelength of a packet is not pre-determined and this packet might be converted to any of the $M$ available wavelengths. Therefore, the tuning range of a full-range WC is equal to the number of wavelengths per fiber $M$.

The architecture presented in Fig. 1 is now described in detail. At the input interfaces, channels are de-multiplexed and those related to the same wavelength on the $N F$ input fibers are connected to a space matrix dedicated to that wavelength (see space stage $A$ ). In space stage $A, M$ space switching matrices (one per wavelength) are employed for two reasons: first, to provide a modular architecture exploiting small switching matrices as opposed to a single large matrix; second, to minimize the number of Optical Gates (OGs), at least when single-stage space switching matrices are considered. Each one of the $M$ matrices manages contentions for a particular wavelength, thus those matrices are all needed and there is not a duplication of switching resources. OGs can be implemented for example by using Semiconductor Optical Amplifiers (SOAs), micro-ring resonators, acousto-optical devices and so on $[1,20]$. Fig. 1 points out that the input fibers are connected to the space matrices in stage $A$ according to a shuffle permutation. Packets arriving at a space matrix can be either directly forwarded to the $N F$ output fibers or forwarded to the $N_{W C}$ WCs. Therefore, the size of the $M$ space matrices at stage $A$ is $N F \times\left(N F+N_{W C}\right)$.
After the WC stage, at space stage $B$, each WC is connected to a $1 \times N F$ space switch needed to connect it to the output fibers, so there are $N_{W C}$ space switches with size $1 \times N F$. This is not the only possible solution, anyway for single stage implementations it requires the lowest number of OGs. A set of $N F$ combiners $N_{W C}: 1$, each connected to one of the output fibers, is used to multiplex the signals coming from the $N_{W C}$ WCs. The $N_{W C}$ space switches and the $N F$ combiners are interconnected according to a shuffle permutation, so that each WC is connected to all combiners, and vice versa. Finally, at the output fibers, NF combiners $(M+1): 1$ are used to multiplex the signals coming directly from switching stage $A$ (up to $M$ ) and the signals coming from the WC pools. Neglecting the signals coming from the WCs, the signals from stage $A$ are connected to the combiners according to a shuffle permutation.

The proposed architecture is equipped with multi-fiber interfaces; this kind of architecture is able to forward up to $F$ packets coming on the same wavelength to the same OI (one packet per fiber), thus partially solving contention among packets on the same wavelength. In the monofiber case $(F=1)$, only one packet per wavelength can be forwarded to the same OI. Assuming a fixed number of channels per interface $N_{C}=M F$, the higher the number of fibers per interface (denoted by $F$ ), the better the packet loss performance would be expected even with no wavelength conversion. Furthermore, when wavelength conversion is considered, increasing $F$ allows to reduce the number of WCs needed in the architecture (contention partially solved by multi-fiber solution) and also to reduce the range of each WC ( $M F$ constant, if $F$ increases $M$ decreases). This aspect will be further investigated in Section 4. On the other hand, increasing the number of fibers per interface also leads to an increase in the complexity of the architecture in terms of optical devices. Therefore, the optimal trade-off needs to be studied to obtain desired performance with minimum complexity.

The complexity of the A-MF-SPN architecture can be evaluated by observing that the space stage $A$ needs $M$ single-stage space switches of size $N F \times\left(N F+N_{W C}\right)$ while space stage $B$ needs $N_{W C}$ single-stage space switches of size $1 \times N F$. Using basic algebra, the overall complexity can be expressed in terms of the overall number of optical gates $N_{O G}^{S P N}$ $N_{O G}^{S P N}=N^{2} F N_{C}+N\left(N_{C}+F\right) N_{W C}$.

Considering the first term, the complexity increases linearly with $F$ when $N_{C}$ is kept fixed. For the second term, the complexity increases with $F$ if the total number of WCs $N_{W C}$ is fixed. However, it is important to emphasize that $N_{W C}$ decreases as $F$ increases (see Section 4) for a fixed desired packet loss probability. Therefore, the dependence of the second term on $F$ needs to be thoroughly evaluated.

\subsection{A-MF-SPIW architecture}

This section introduces the A-MF-SPIW architecture (Fig. 2) considering again $N$ input/output interfaces (II/OIs) each provided with $F$ optical fibers carrying $M$ wavelengths. The total number of channels per interface is $N_{C}=M F$. To solve contention in wavelength domain, the architecture is 


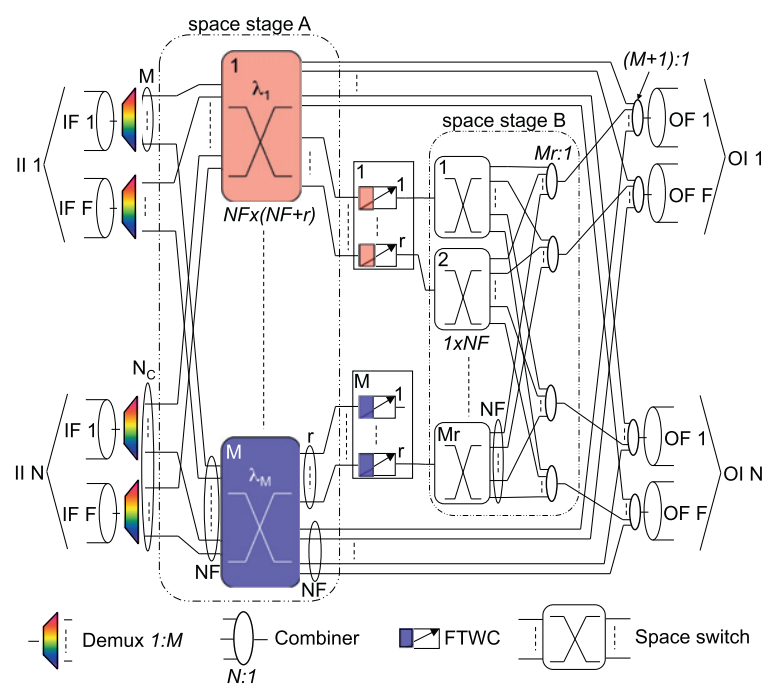

Fig. 2. A-MF-SPIW architecture with $N$ input/output interfaces equipped with $F$ optical fibers carrying $M$ wavelengths each. The switch is equipped with $M$ pools, each of them with $r(<N F)$ WCs dedicated per input wavelength.

equipped with fixed-input/tunable-output WCs which are simpler and less expensive than tunable-input/tunable-output ones [6,9]. WCs are partitioned in $M$ pools of $r(<N F)$ WCs each; each pool serves packets coming on the same wavelength, hence the WCs in a given pool are shared-perinput-wavelength [10]. The total number of WCs is $N_{W C}=M r$ and their output range is $M$.

The same structure introduced for the A-MF-SPN is maintained. Consequently, at space stage $A, M$ space matrices interconnect IIs, OIs and WCs. The real advantage of the A-MF-SPIW architecture compared to the A-MF-SPN comes from the shared-per-input-wavelength scheme itself; only the channels related to a given wavelength need to be connected to the corresponding WC pool, which indeed uses fixed-input WCs. For this reason, a space matrix related to a given wavelength is connected to just $r$ WCs, and not to all $N_{W C}$ WCs as in A-MF-SPN (compare the output of space stage $A$ in Figs. 1 and 2). The space stage $B$ is exactly the same as for A-MFSPN. Consequently, even though the SPIW sharing scheme requires in general more WCs than SPN, SPIW provides certain advantages in terms of the number of OGs required, as will be evidenced in Section 4.3.

The complexity of the A-MF-SPIW architecture can be evaluated by taking into account that the space stage $A$ needs $M$ single-stage space switches of size $N F \times(N F+r)$ while the space stage $B$ needs $N_{W C}$ single-stage space switches of size $1 \times N F$. After some math (recalling $N_{C}=M F$ and $N_{W C}=M r$ ), the complexity in terms of the number of optical gates can be expressed as

$N_{O G}^{S P I W}=N^{2} F N_{C}+2 N F N_{W C}$

Considering the first term, the complexity increases linearly with $F$ when $N_{C}$ is kept fixed. For the second term, the complexity increases linearly with $F$ if the total number of WCs $N_{W C}$ is fixed. It is anyway important to emphasize that $N_{W C}$ decreases as $F$ increases (see Section 4 ) for a fixed desired loss probability. A qualitative analysis of the complexity can be described as follows: in space stage $A$, the size of each space switch increases as $(N F)^{2}+N F r=F^{2}\left(N^{2}+\left(N / N_{C}\right) N_{W C}\right)$, but the number of space switches $\left(M=N_{C} / F\right)$ is in inverse relationship with $F$. In space stage $B$, the size of each space switch increases as $N F$, and the number of space switches is related to $N_{W C}$, which decreases as $F$ increases.

Both architectures are operated in an asynchronous scenario, where a packet is scheduled as it arrives at the input interface. The packet is forwarded without conversion as first choice. With this purpose, when a packet arrives at the switch on wavelength $w$, the scheduling algorithm randomly selects one fiber (say $f$ ) on the destination interface for which wavelength $w$ is available and then the packet is forwarded to wavelength $w$ of fiber $f$. If all the wavelength channels related to $w$ are busy then another outgoing wavelength (say $g$ ) is selected randomly among the ones that are free on at least one fiber. One of such output fibers (say $h$ ), where wavelength $g$ is available, is randomly selected and the packet is forwarded on wavelength $g$ of fiber $h$ using an available WC. For A-MFSPN, this means one of the $N_{W C}$ WCs, no matter which one, while for the A-MF-SPIW this means one of the $r$ WCs in the pool dedicated to input wavelength $w$. A packet is lost either when all $N_{C}$ channels on the destination interface are busy at the time the packet arrives (output blocking) or when the arriving packet requires conversion and all $N_{W C}(\mathrm{SPN})$ or $r$ (SPIW) WCs are busy (converter unavailability). More complex scheduling policies are left for future research. The analytical models presented in Section 3 are developed taking into consideration the scheduling procedure described above.

\section{State aggregation-based analytical model of A-MF-SPIW and A-MF-SPN}

In the proposed model, the traffic destined to OI $n$ $(n=1, \ldots, N)$, is assumed to be a Poisson process with intensity $\lambda^{(n)}$ and overall traffic density is denoted by $\lambda=\sum_{n=1}^{N} \lambda^{(n)}$. The following traffic intensity pattern is used for numerical evaluation:

$\lambda^{(n)}=\lambda \frac{1-f}{1-f^{N}} f^{n-1}, \quad 1 \leq n \leq N$,

where $f \geq 1$ is called the traffic imbalance parameter $[7,8]$. The traffic tends to get more asymmetric across destination OIs as the parameter $f$ increases, whereas in the case $f \rightarrow 1$ the traffic tends to be symmetric over all OIs. The degree of traffic asymmetry also depends on $N$, i.e., with the same value of $f$, the traffic gets more asymmetric for larger $N$. The utilization of a single-parameter traffic asymmetry model given in formula (3) is crucial to study the impact of traffic imbalance on the performance of the switch under different configurations. The wavelength of an incoming optical packet is also assumed to be uniformly distributed over the $M$ wavelengths, i.e., balanced traffic across incoming wavelengths. This is because the A-MF-SPIW shares WCs among the different wavelengths, so for fairness reason the traffic should be balanced among the wavelengths. 
Otherwise, the most loaded wavelengths would experience a higher packet loss, or the number of WCs dedicated to those wavelengths should be increased. Packet lengths are assumed to be exponentially distributed with average set to unity $(\mu=1)$, thus it represents the time unit in this paper. The load per output wavelength channel in the balanced traffic case is denoted by $p=\lambda /(N M F)$. Even though in the imbalanced traffic case the average load per output wavelength differs from one interface to another, $p$ is still considered as the conventional load per output wavelength when presenting results in the imbalanced traffic case.

First, the analytical model for A-MF-SPIW is described and then the simple modification required to extend the model to the A-MF-SPN case is explained. Let us define $X_{n}^{j}(t)$ $\left(0 \leq X_{n}^{j}(t) \leq F\right)$ as the number of occupied output channels using the $j$ th wavelength corresponding to OI $n$ for $1 \leq j \leq M, 1 \leq n \leq N$. Also let $Y_{l}(t)$ denote the number of WCs occupied at the wavelength converter pool $l$ for $1 \leq l \leq M$ for SPIW. From the description of the problem, it is not difficult to show for SPIW that the multi-dimensional process $\left\{X_{n}^{j}(t), Y_{l}(t): 1 \leq j \leq M, 1 \leq n \leq N, 1 \leq l \leq M\right\}$ is Markov. However, the corresponding Markov chain is so large rendering any computation infeasible, a phenomenon known as "curse of dimensionality". Therefore, there is a need for approximative techniques. For a fixed OI $n$, the state aggregation technique is proposed, by aggregating the states of the process $\left\{X_{n}^{j}(t): 1 \leq j \leq M\right\}$ into a new aggregated process $\left\{L^{(n)}(t)\right\}$ which denotes the number of occupied wavelength channels for OI $n$ at time $t$. The former process has $(F+1)^{M}$ states whereas the aggregated process has only $N_{C}+1=$ $M F+1$ states. Therefore, a potential for significant statespace reduction is imminent. For the purpose of aggregation, the following two assumptions hold:

- When $L^{(n)}(t)=i$, the probability that an arriving packet at time $t$ requires conversion is approximated by a quantity $p_{i}^{(n)}, i=0,1, \ldots, N_{C}-1$, that only depends on $i$ and $n$. When $i=N_{c}$, all wavelength channels on output fiber $n$ are busy, hence $p_{N_{c}}^{(n)}=0$, since an arriving packet can not be forwarded thus it does not require conversion. This probability actually depends on $n$ and also how the $i$ occupied channels are distributed over the $M$ wavelengths and not on the value $i$ only. One of the goals of this paper is to assess if this approximation (for the sake of computation) effectively models the system of interest.

- Given that a packet requires conversion, the probability of this packet finding all converters busy in its associated pool is given by the quantity $p_{B}$ and this probability is assumed to be independent of the state of $L^{(n)}(t)$ at the epoch of packet arrival. This approximation is valid for relatively large $N$ since the contribution of one single OI $n$ on the aggregate behavior of the converter pool diminishes for larger $N$ as evidenced in our earlier work for the mono-fiber case [7].

Under these two assumptions, the random process $\left\{L^{(n)}(t)\right\}$ becomes a non-homogeneous Birth-Death (BD) type Markov chain with birth rate $\eta_{i}^{(n)}$ at state $i$ (the transition rate from state $i$ to $i+1)$ which is written as

$\eta_{i}^{(n)}=\lambda^{(n)}\left[\left(1-p_{i}^{(n)}\right)+p_{i}^{(n)}\left(1-p_{B}\right)\right], \quad i=0, \ldots, N_{C}-1$.

On the other hand, death rate at state $i$ (transition rate from state $i$ to $i-1$ ) is given without loss of generality by $\mu_{i}^{(n)}=\mu i=i$ since the time unit is equal to the average packet duration.

If the probabilities $p_{i}^{(n)}$ and $p_{B}$ were known, then the steady-state probabilities $\pi_{i}^{(n)}\left(i=0,1, \ldots, N_{C}\right)$ could be found via

$\pi_{i}^{(n)}=\lim _{t \rightarrow \infty} P\left(L^{(n)}(t)=i\right)=\prod_{k=0}^{i-1} \frac{\eta_{k}^{(n)}}{\mu_{k+1}^{(n)}} \pi_{0}^{(n)}, \quad 1 \leq i \leq N_{C}$,

where $\pi_{0}^{(n)}$ comes from the normalization condition: all probabilities should sum to unity. Using the "Poisson Arrivals See Time Averages" (PASTA) principle, the overall Packet Loss Probability (PLP) for an arbitrary packet would then be given by

$P L P=\frac{1}{\lambda}\left[\sum_{n=1}^{N} \pi_{N_{C}}^{(n)} \lambda^{(n)}+\sum_{n=1}^{N} \sum_{i=1}^{N_{C}-1} \lambda^{(n)} \pi_{i}^{(n)} p_{i}^{(n)} p_{B}\right]$.

The first term amounts to the case when an arbitrary arriving packet finds all output wavelength channels occupied at its destination OI. On the other hand, the second term considers the case when there are idle channels on OI $n$ but the packet's conversion requirement is not satisfied due to the lack of an appropriate WC.

The analysis carried out so far applies to both A-MFSPIW and A-MF-SPN architectures. A novel scheme is now proposed to obtain the quantities $p_{B}$ and $p_{i}^{(n)}$ for both architectures. Let us start with the A-MF-SPIW architecture to find the quantity $p_{B}$ assuming that $p_{i}^{(n)}$ as known. Note that the intensity of traffic destined to OI $n$ requiring conversion is given by

$v^{(n)}=\sum_{i=1}^{N_{C}-1} \lambda^{(n)} \pi_{i}^{(n)} p_{i}^{(n)}$

In the case of A-MF-SPIW, the intensity of the overall traffic destined to a WC pool dedicated to any one of the wavelengths is denoted by $v_{S P I W}$ which is given by

$v_{\text {SPIW }}=\frac{\sum_{n=1}^{N} v^{(n)}}{M}$,

since there are $N$ such OIs and the overall traffic is uniformly distributed among $M$ WC pools. Under the assumption that this traffic is Poisson, the quantity $p_{B}$ can be obtained using the Erlang-B formula corresponding to the case of $r$ servers fed with Poisson traffic with intensity $v_{\text {SPIW }}$.

For the purpose of approximating the quantity $p_{i}^{(n)}$, the following observation is relevant. In the case of no wavelength conversion, i.e., $r=0$, the quantities $p_{i}^{(n)}$ can be explicitly found such that the loss probability using (6) is exact. Note that exact solutions are easily obtainable in case $r=0$, as presented in the following section. Since the quantity $p_{i}^{(n)}$ in our assumption does not depend on $r$, we suggest that the quantity $p_{i}^{(n)}$ (that is explicitly found for the case $r=0$ ) is to be used in calculations involving other values of $r$. Let us now focus on the case $r=0$ in which 
case for OI $n, M$ server groups each one with $F$ servers are present and fed with Poisson traffic with intensity $\lambda^{(n)} / M$. Let $y_{l}^{(n)}$ denote the steady-state probability that an arbitrary wavelength $k, 1 \leq k \leq M$ is occupied on $l, 0 \leq l \leq F$ fibers on OI $n$. Actually, $y_{l}^{(n)}$ does not depend on the particular wavelength $k$

$y_{l}^{(n)}=\prod_{m=0}^{l-1} \frac{\lambda^{(n)}}{M \mu_{m+1}^{(n)}} y_{0}^{(n)}, \quad 1 \leq l \leq F$,

where $y_{0}^{(n)}$ is again obtained using the normalization condition. Let $x_{j}^{(n)}$ denote the steady-state probability of $j, 0 \leq j \leq N_{C}$ channels being occupied for OI $n$ when $r=0$. The quantity $x_{j}^{(n)}$ can be obtained using an $M$-fold convolution of $y_{l}^{(n)}$

$x_{j}^{(n)}=\underbrace{\sum_{l_{1}=0}^{F} \sum_{l_{2}=0}^{F} \cdots \sum_{l_{M}=0}^{F}}_{j=l_{1}+l_{2}+\cdots+l_{M}} \prod_{i=0}^{M} y_{l_{i}}^{(n)}$.

Now, consider the following birth-death process $X^{(n)}(t)$ with death rate at state $j$ being equal to $j$ and birth rate $\beta_{j}^{(n)}$ expressed as:

$\beta_{j}^{(n)}=\frac{(j+1) x_{j+1}^{(n)}}{x_{j}^{(n)}}, \quad j=0,1, \ldots, N_{C}-1$.

It is clear from the global balance equations of this Markov chain that $x_{j}^{(n)}$ is the steady-state probability $\lim _{t \rightarrow \infty} P\left(X^{(n)}(t)=j\right)$. When $r=0$, the one-dimensional Markov chain $\left\{X^{(n)}(t)\right\}$ constructed above using $x_{j}^{(n)}$ as its birth rates has the steady-state probabilities $x_{j}^{(n)}$ and therefore can be used to exactly characterize the system behavior when $r=0$. In this paper, $p_{i}^{(n)}$ is chosen such that the birth rates of the two Markov chains corresponding to $L^{(n)}(t)$ and $X^{(n)}(t)$ are the same when $r=0$ and thus $p_{B}=1$ (loss due to lack of WCs). By comparing (4) and (11) with $p_{B}=1$, $\lambda^{(n)}\left(1-p_{i}^{(n)}\right)=\beta_{i}^{(n)}$ is obtained or equivalently

$p_{i}^{(n)}=1-\frac{\beta_{i}^{(n)}}{\lambda^{(n)}}, \quad i=0,1, \ldots, N_{C}-1$.

By choosing $p_{i}^{(n)}$ in this way, a one-dimensional Markov chain $L^{(n)}(t)$ is applied to correctly find the loss probabilities for the case $r=0$. For the case $r>0$, the same expression (12) to write $p_{i}^{(n)}$ is used. Note from (4) that as $r \rightarrow \infty$ then $p_{B} \rightarrow 0$ and the process $L^{(n)}(t)$ approaches to an Erlang loss system with $N_{C}$ servers offered with Poisson traffic with intensity $\lambda^{(n)}$ and the approximative approach presented in (12) therefore becomes also exact in this regime. Consequently, the approach of using (12) for conversion requirement probabilities provides exact results in both ends of the spectrum for $r$, namely for cases in which $r=0$ and $r \rightarrow \infty$. The main principle underlying our approximative approach is to use (12) throughout the entire spectrum for $r$.

Note that in the above procedure $p_{B}$ is evaluated using a fixed-point procedure. To summarize this procedure, first $p_{i}^{(n)}$ is obtained as given in (12). Then the fixed-point iterative process is started with an arbitrary initial value for $p_{B}$ to construct the Markov chain for the process $\left\{L^{(n)}(t)\right\}$ for each $n$. The corresponding Markov chain is then solved to find the probabilities $\pi_{i}^{(n)}$, and then the probability $p_{B}$ is re-calculated as the loss probability of an Erlang loss system with $r$ servers considering Poisson traffic with intensity $v_{S P I W}$, defined in formula (8). The overall loss probability PLP is obtained by the formula (6). This procedure is then repeated until convergence, i.e., until two successive values for PLP are as close to each other as desired. Once the fixed-point iterations converge, PLP is used as an approximation for the packet loss probability of the switch.

On the other hand, for the case of A-MF-SPN, the only part of the algorithm that requires modification is the part where $p_{B}$ is obtained. For this purpose, the intensity of overall traffic destined to the shared-per-node WC pool is denoted by $v_{S P N}$ and is given by

$v_{S P N}=\sum_{n=1}^{N} v^{(n)}$.

The quantity $p_{B}$ is then found using the Erlang-B formula corresponding to the case of $N_{W C}$ servers offered with Poisson traffic with intensity $v_{S P N}$. All other parts of the proposed model remain the same as for the A-MF-SPIW scheme.

\section{Numerical results}

This section presents: the validation of the proposed models comparing analytical and simulation results (Section 4.1); the comparison between A-MF-SPIW and A-MF-SPN in terms of packet loss (Section 4.2) using only the analytical model; the complexity analysis and comparison between the two architectures (Section 4.3) again using the analytical model.

\subsection{Validation of the analytical models}

Simulation results have been obtained by applying the random scheduling procedure described in Section 2 to Poisson arrivals. The confidence interval of the simulations is less than or equal to $10 \%$ of the average PLP, with 95\% probability. To provide a fair comparison between architectures, the quantity called Conversion Ratio, $C R=\left(N_{W C} / N N_{C}\right)=r /(N F)$, is introduced. The range of values for $C R$ is between 0 (no conversion case) and 1 (one WC per channel). It is worthwhile noting that for the A-MF-SPIW architecture, only some discrete values of $C R$ are allowed; in fact, each time a WC per wavelength is added (i.e., $r$ increases by one unit), $N_{W C}$ increases by $M$ units (Section 2.2).

Fig. 3(a) and (b) presents model validation in the mono-fiber case $(F=1)$ for A-MF-SPN and A-MF-SPIW, respectively. Analytical $(A)$ and simulation $(S)$ results are compared. PLP is presented as a function of $C R$ as in the case $N=32, N_{C}=M F=16, F=1$ and load $p$ varying from 0.2 to 0.8 . The figures point out how the analysis is very close to simulation for both A-MF-SPN and A-MF-SPIW. 
a

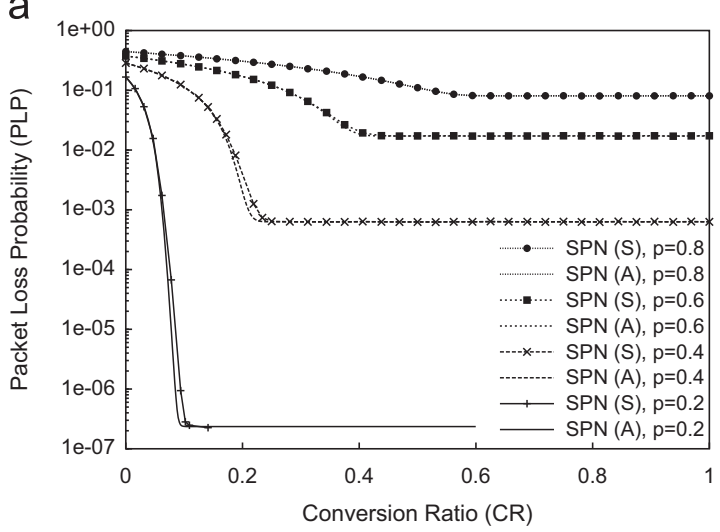

b

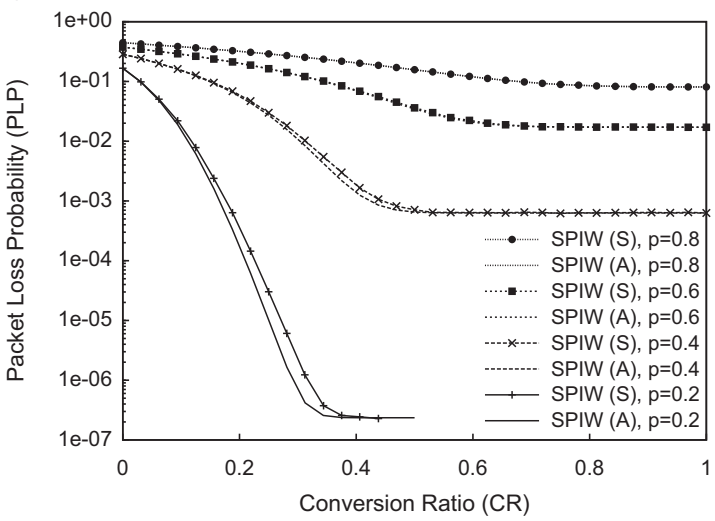

Fig. 3. Comparison between analytical $(A)$ and simulation $(S)$ results for A-MF-SPN (a) and A-MF-SPIW (b). PLP is presented as a function of the conversion ratio $C R$, as in the case $N=32, N_{C}=M F=16, F=1$ (monofiber case) and load $p$ varying from 0.2 to 0.8 .

In the case of A-MF-SPIW, the analytical model slightly underestimates the PLP, especially when the load and PLP are low. Indeed, at light loads, the approximation in loss evaluation at the WC pools influences the overall loss more significantly. Both models provide anyway good results and they exactly capture the asymptotic values of the PLP, which are only related to output blocking. Moreover, the value of $\mathrm{CR}$ at which the asymptotic value of the PLP is attained, is also well approximated by the analytical model, which returns slightly optimistic values.

Fig. 4(a) and (b) depicts the same scenario for the multi-fiber case. The PLP is plotted as in the case $N=32$, $N_{C}=M F=16, F=4$ with load $p$ varying from 0.2 to 0.8 . For the multi-fiber $F=4$ case, the models provide also remarkably accurate results compared to simulations. Hence, the models are able to capture the role of the multi-fiber solution in solving contentions among packets in the space domain by exploiting wavelength reuse in different fibers. It is also possible to see how the number of WCs needed to reach the asymptotic value of the PLP is lower when $F=4$ compared with the previous case $F=1$ (Fig. 3), proving that the multi-fiber solution allows to substantially reduce the number of WCs and their range, since the number of wavelengths is $M=N_{C} / F=4$ as opposed to $M=16(F=1$ case $)$.

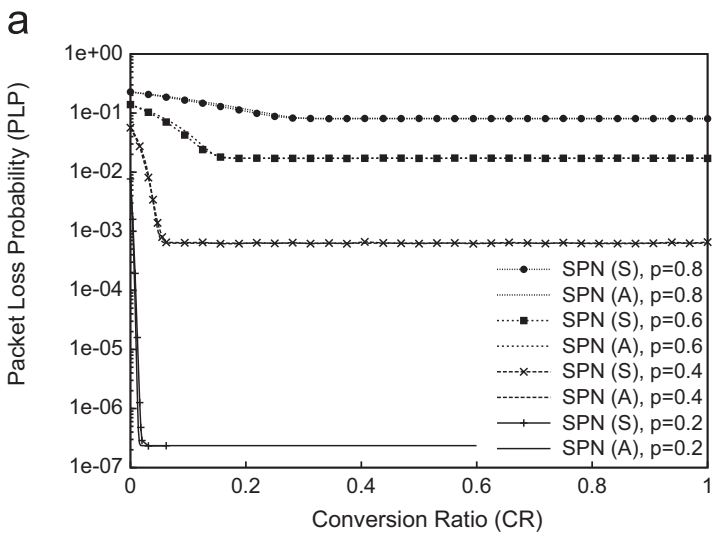

b

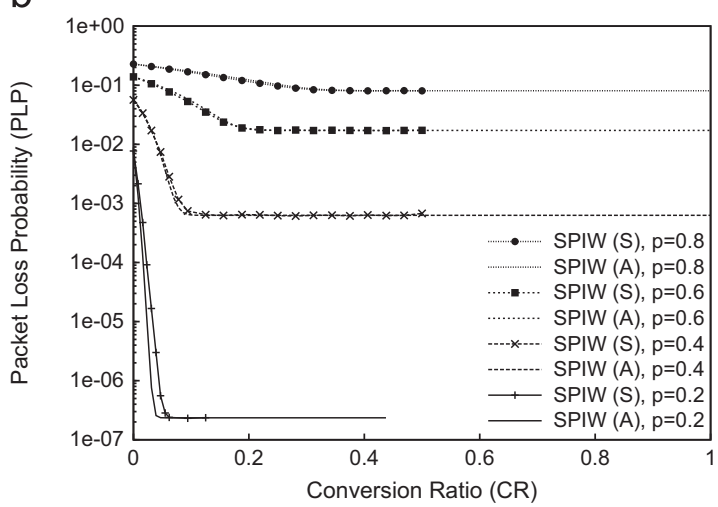

Fig. 4. Comparison between analytical (A) and simulation (S) results for A-MF-SPN (a) and A-MF-SPIW (b). PLP is presented as a function of the conversion ratio $C R$, as in the case $N=32, N_{C}=M F=16, F=4$ (multi-fiber case) and load $p$ varying from 0.2 to 0.8 .

This observation is even more evident in Fig. 5(a) and (b) depicting the PLP as a function of $C R$ as in the case $N=32, N_{C}=M F=16$, load $p=0.3$ and $F$ varying from 1 (mono-fiber) to 8 . These figures clearly show the advantage of the multi-fiber solution as the number of fibers per interface $F$ increases. As a matter of fact, the asymptotic value of the PLP is reached with a reduced number of WCs for increasing $F$, and the saving that can be achieved is remarkable. It is also interesting to observe that the models perform better when the number of fibers increases. Therefore, the model is well-suited for capturing the multi-fiber effect.

To further validate the models under different number of II/OIs and channels per interface $N_{C}$, Fig. 6(a) and (b) plots the PLP in the case $N=16, N_{C}=M F=32, F=1$ (mono-fiber case) and load $p$ varying from 0.4 to 0.7 , while Fig. $7(a)$ and (b) considers the multi-fiber case $N=16, N_{C}=M F=32, F=4$ and load $p$ varying from 0.4 to 0.7. It can be observed how the model for the A-MFSPN provides accurate results for this scenario for both mono-fiber and multi-fiber cases, while the model for A-MF-SPIW is less precise especially when the load is low. The model works perfectly for low values of $C R$ based on the fact that the state-aggregation scheme is exact when no converters are used. When the load is low for A-MFSPIW and for higher values of $C R$, OIs and converter pools 
a

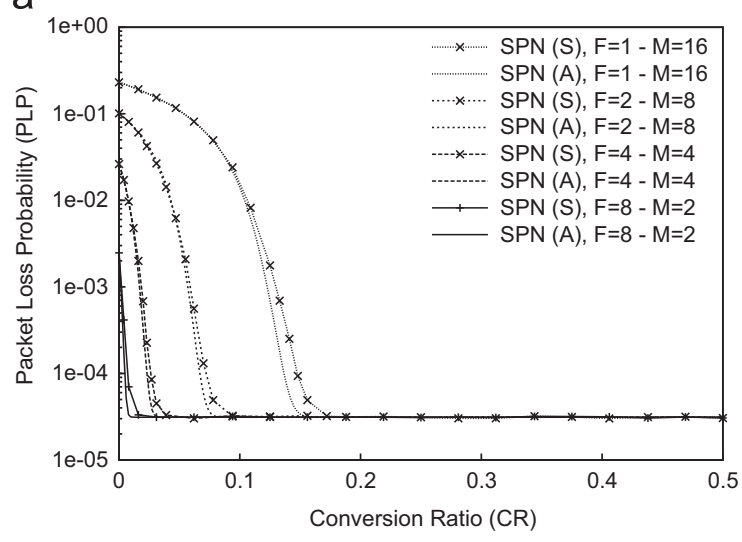

b

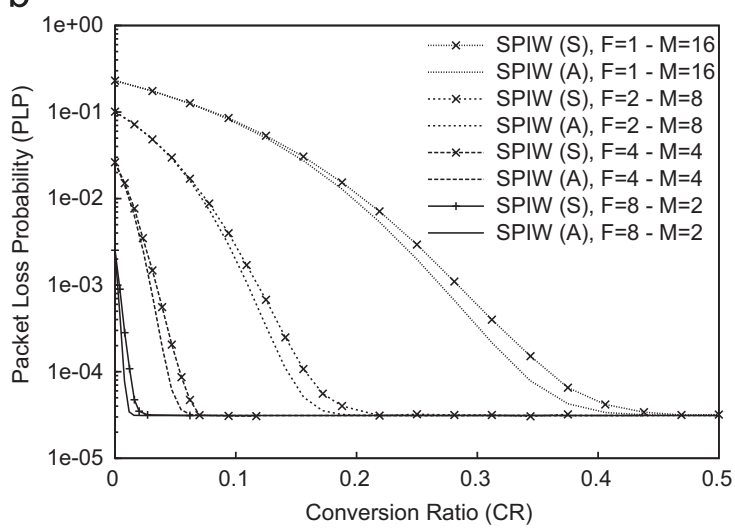

Fig. 5. Comparison between analytical $(A)$ and simulation $(S)$ results for A-MF-SPN (a) and A-MF-SPIW (b). PLP is presented as a function of the conversion ratio $C R$, as in the case $N=32, N_{C}=M F=16$, load $p=0.3$ and $F$ varying from 1 (mono-fiber) to 8 .

turn out to be more correlated, a situation which is not captured by the analytical model. This situation is emphasized for relatively smaller switch sizes $N$ and larger number of wavelengths $M$. In any case, the proposed computationally efficient model captures very well the general behavior of the PLP for a wide range of scenarios. It can be used to find the limit value of $C R$ above which no more loss probability reduction is possible, i.e., asymptotic loss probability is attained. Observe that when the model for the A-MF-SPIW is used to estimate the number of WCs needed to reach asymptotic loss, the actual loss (indicated by simulation) would be higher than the asymptotic one, but in the same order of magnitude. This is true even when the discrepancy between analysis and simulation for A-MF-SPIW is relevant in the region where the loss rapidly decreases (Figs. 6(b) and 7(b)).

Comparison between analysis and simulation is presented for imbalanced traffic in Fig. 8(a) and (b), for A-MFSPN and A-MF-SPIW, respectively. PLP is plotted as a function of $C R$, as in the case $N=32, N_{C}=M F=16, F=2$ (multi-fiber case), load $p=0.2$ and imbalance parameter $f=1.00,1.01,1.05$ and 1.10 . Both figures highlight that the models capture the effects related to imbalanced traffic profile, providing accurate approximations of PLP. The PLP a

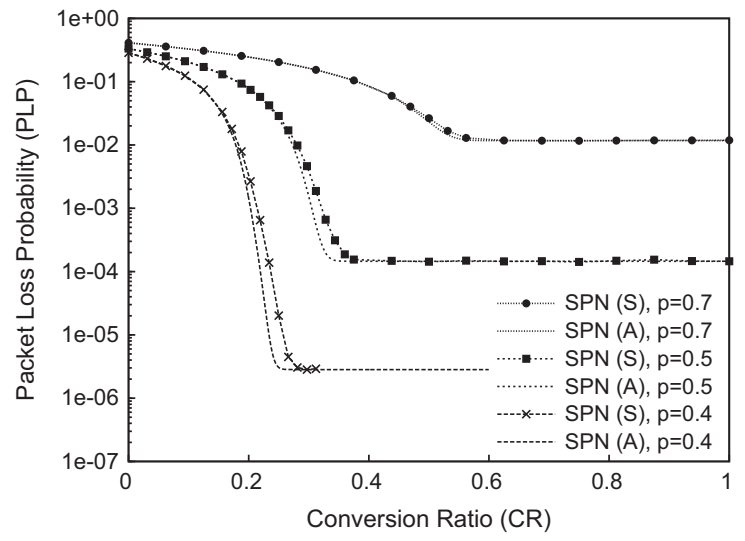

b

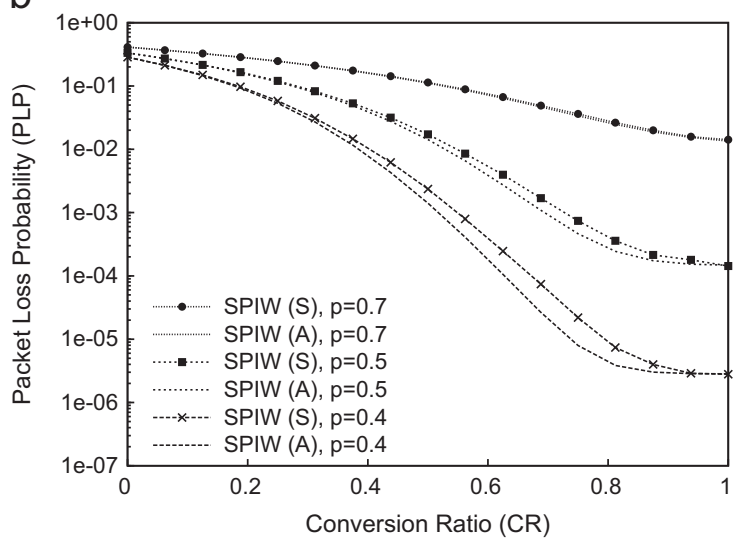

Fig. 6. Comparison between analytical $(A)$ and simulation $(S)$ results for A-MF-SPN (a) and A-MF-SPIW (b). PLP is presented as a function of the conversion ratio $C R$ in the case $N=16, N_{C}=M F=32, F=1$ (mono-fiber case) and load $p$ varying from 0.4 to 0.7 .

rapidly increases as $f$ increases due to the fact that some of the OIs are relatively more loaded than the others, especially in scenarios when $N$ is high. As a matter of fact, the imbalance traffic intensities are related to the number of interfaces $N$ by observing formula 3 in Section 3 . Indeed, being the imbalance traffic intensities proportional to $f^{n-1}$ in formula 3 , when $N$ is large, the OI $N$ will be quite loaded due to the high value of $N$, compared to the others, leading to very high loss. This effect is captured by the models, which can then be applied even for imbalanced scenarios.

\subsection{Performance comparison of A-MF-SPN and A-MF-SPIW}

This section compares the proposed architectures in terms of PLP using analytical results only since it has been proven in the previous section that analytical models provide accurate approximations of PLP. In this section graphs plot packet loss for A-MF-SPIW and A-MF-SPN together, thus providing a direct comparison.

Figs. 9(a) and (b) compare A-MF-SPN and A-MF-SPIW architectures in mono-fiber and multi-fiber cases, respectively. In particular, PLP is presented as a function of the 
a

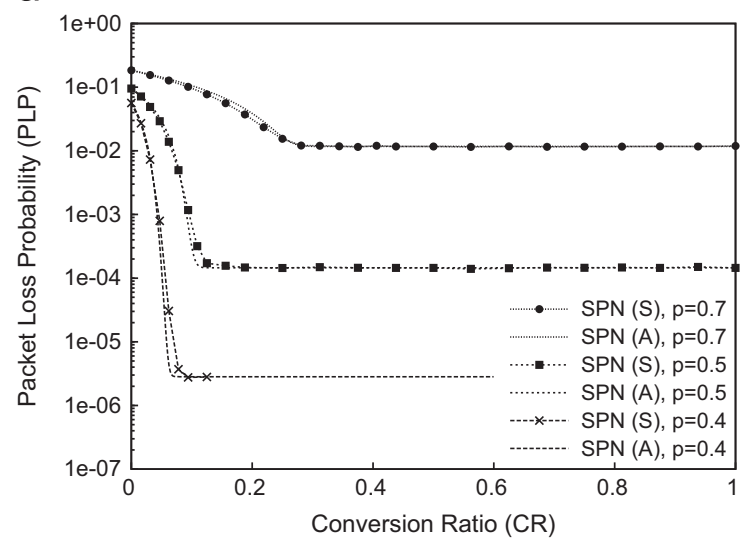

b

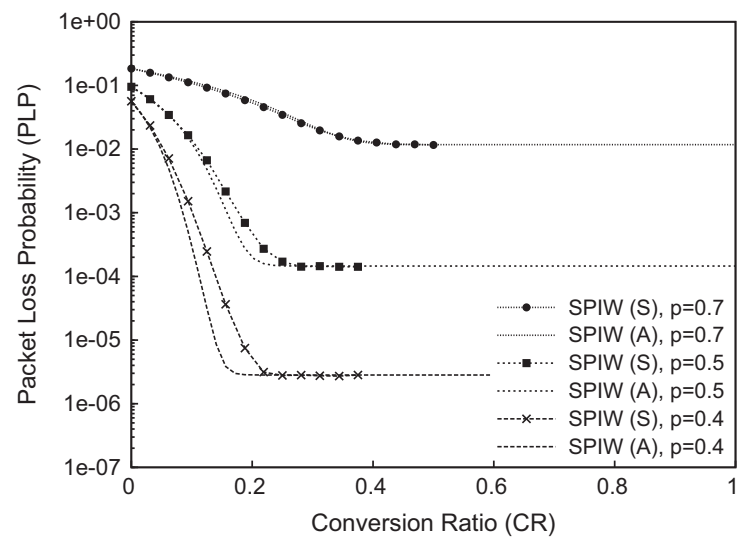

Fig. 7. Comparison between analytical (A) and simulation (S) results for A-MF-SPN (a) and A-MF-SPIW (b). PLP is presented as a function of the conversion ratio $C R$, as in the case $N=16, N_{C}=M F=32, F=4$ (multi-fiber case) and load $p$ varying from 0.4 to 0.7 .

conversion ratio $C R$ varying load $p$ from 0.2 to 0.8 as in the case $N=32, N_{C}=M F=16, F=1$ (a) and $F=4$ (b). Both architectures provide the same asymptotic PLP, which is indeed related to output blocking only, and it is not related to the sharing scheme. The results clearly demonstrate how in the mono-fiber case the A-MF-SPN allows to reach the asymptotic value of PLP with a fewer number of WCs compared to A-MF-SPIW, especially when the load in low, allowing to save a substantial number of WCs and thus reduce the overall cost related to WC use. Nevertheless, there is marginal difference between the two architectures in the multi-fiber case, given that in this case both architectures allow to reach the asymptote with a relatively small number of the WC stages. This also highlights that the A-MF-SPIW performance greatly improves as the number of fibers $F$ increases (compare 9(a) and (b) for SPIW).

Figs. 10(a) and (b) confirm the improvement of the AMF-SPIW as $F$ increases by presenting the PLP in case the number of fibers per interface is varied, for two different scenarios. PLP is presented as a function of the conversion ratio $C R, F$ varying between 1 and 8 , in the cases: (a) $N=32, N_{C}=M F=16, p=0.3$ and (b) $N=16, N_{C}=M F=32$, a

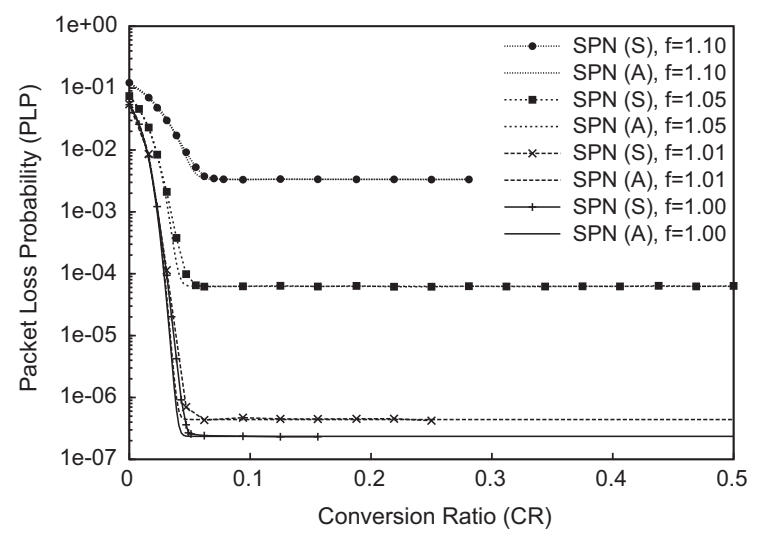

b

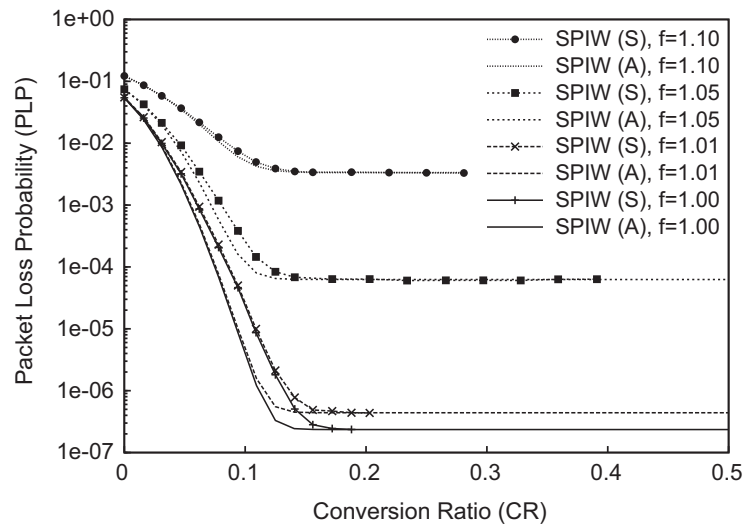

Fig. 8. Comparison between analytical $(A)$ and simulation $(S)$ results for A-MF-SPN (a) and A-MF-SPIW (b). PLP is presented as a function of the conversion ratio $C R$, as in the case $N=32, N_{C}=M F=16, F=2$ (multi-fiber case), load $p=0.2$ and varying the imbalance parameter $f=1.00,1.01$, 1.05 and 1.10 .

$p=0.45$. Both figures highlight how A-MF-SPN and A-MFSPIW performance improves as $F$ increases; this improvement is particularly more emphasized for the A-MF-SPIW and in figure $10(\mathrm{~b})$ where A-MF-SPIW needs a very high number of WCs in the mono-fiber case $(C R>0.8)$. Under this configuration where $M$ is relatively large compared to $N$, the A-MF-SPIW sharing scheme for mono-fiber case is less efficient; WCs are partitioned in too many pools $(M=32)$ each sharing few $(N=16)$ channels and the $C R$ needed to reach the asymptote is very high. This effect has been presented in previous papers for the mono-fiber case and it has been proven that SPIW scheme is more efficient for high $N[7,21]$. However, these new results illustrate how the multi-fiber solution alleviates this problem even with $F=2$, allowing more than $50 \%$ reduction in the number of WCs for both Fig. 10(a) and (b). For SPN, the improvement due to increasing $F$ is more limited, considering that even the mono-fiber case reaches the asymptote with few WCs.

To complete the comparison, Figs. 11(a) and (b) depict the PLP varying the number of channels per fiber $M$ for mono-fiber and multi-fiber cases, respectively (as a consequence $N_{C}$ varies accordingly). PLP is presented as a 
a

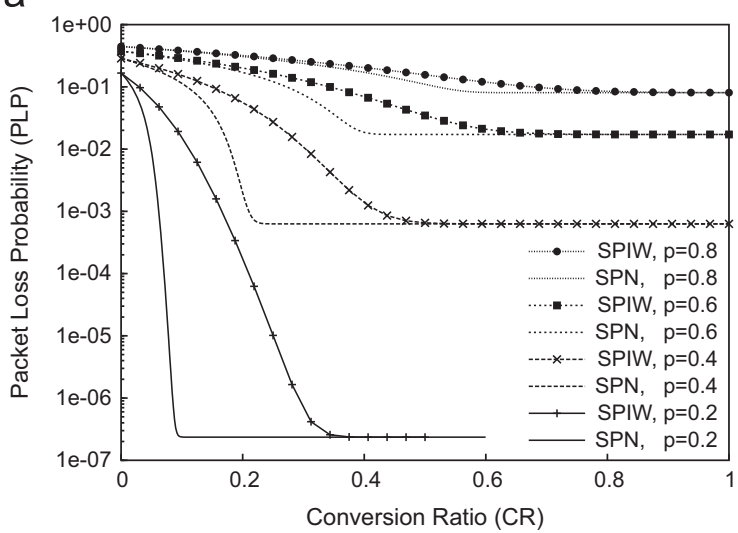

b

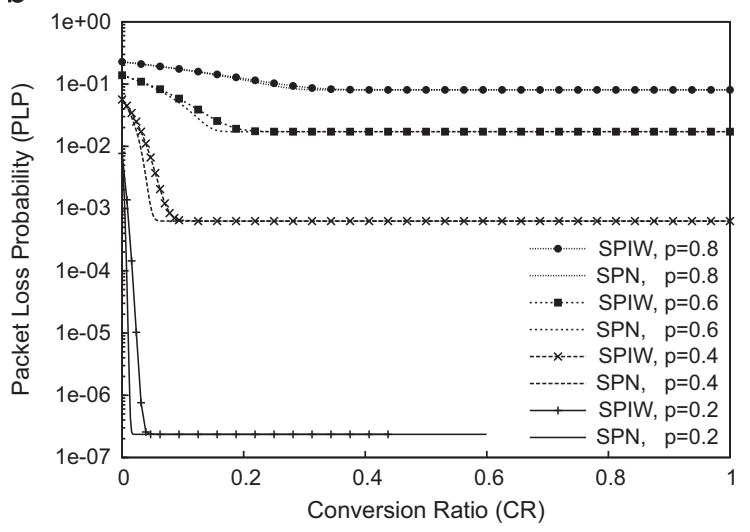

Fig. 9. Comparison between A-MF-SPN and A-MF-SPIW in the monofiber (a) and multi-fiber (b) cases. PLP is presented as a function of the conversion ratio $C R$ varying load $p$ from 0.2 to 0.8 as in the case $N=32$, $N_{C}=M F=16$ and $F=1(\mathrm{a}), F=4$ (b)

function of the conversion ratio $C R$ varying $M(16,24,32)$ as in the case $N=16, p=0.4$ and $F=1$ (a), $F=2$ (b). The figures show how the asymptotic value of PLP moves to lower values as $M$ increases due to the statistical multiplexing gain, providing much better performance with larger $M$. When the architectures are not provided with a sufficient number of WCs, the PLP is the same for all values of $M$, because in this case the PLP is dominated by the loss due to the lack of WCs. However, as $C R$ increases the PLP moves to different asymptotic values for different values of $M$. The threshold value of $C R$ needed to reach the asymptote is much lower for the multi-fiber case, especially for SPIW.

Figs. 12(a) and (b) plot the PLP varying the number of II/OIs, $N$. PLP is presented as a function of the conversion ratio $C R$, varying $N(N=4,8,16)$ as in the case $N_{C}=M F=24, p=0.3$ and $F=1(\mathrm{a}), F=3$ (b). The first figure confirms that in mono-fiber case, A-MF-SPIW provides very poor performance when $N$ is small, as described above, while the performance rapidly improves as $N$ increases. The A-MF-SPN is able to provide good results for all values of $N$; even in this case performance is slightly better with increased $N$. Once again the multifiber solution provides remarkable improvement for the
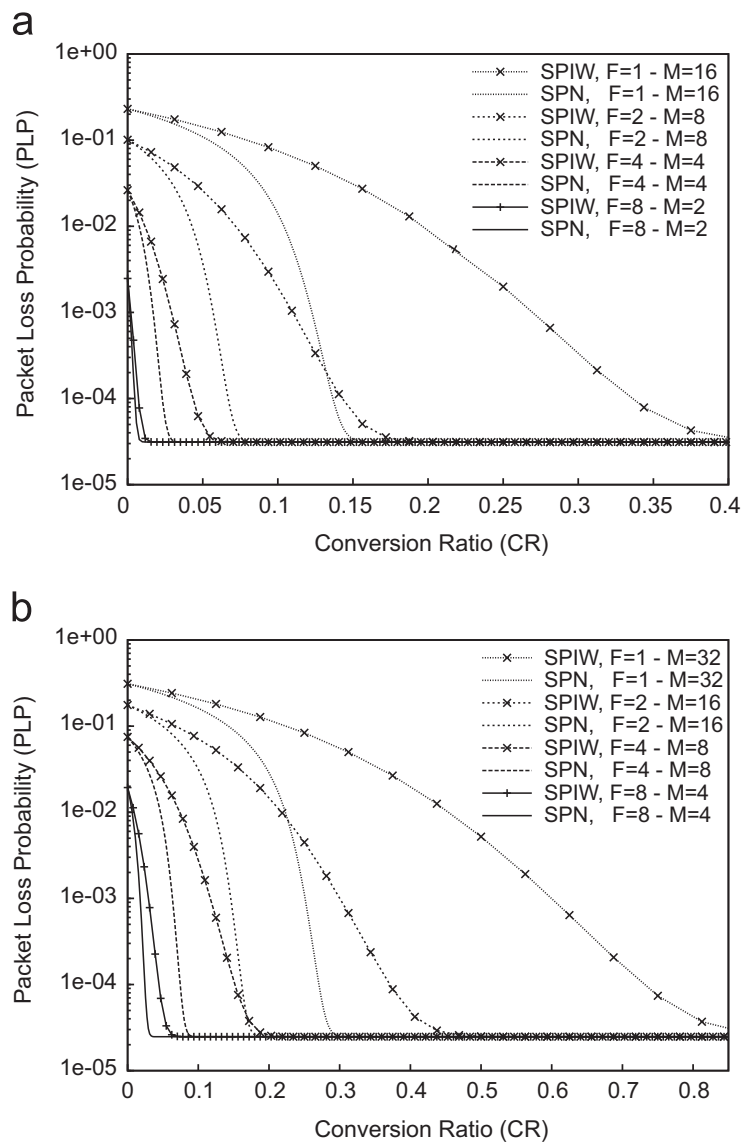

Fig. 10. Comparison between A-MF-SPN and A-MF-SPIW varying the number of fibers per interface. PLP is presented as a function of the conversion ratio $C R$ varying $F$ between 1 and 8 as in the case: (a) $N=32$, $N_{C}=M F=16, p=0.3$ and (b) $N=16, N_{C}=M F=32, p=0.45$.

A-MF-SPIW architecture and the gap between the two architectures become less evident not only with increased $F$ but also with increased $N$.

Figs. 13(a) and (b) show the achievable throughput that can be obtained by fixing a target PLP $<10 \mathrm{e}-4$, for A-MF-SPN and A-MF-SPIW, respectively. Throughput is presented as a function of the number of fibers per interface, $F$, varying the conversion ratio $C R$ in the case $N=16, N_{C}=32$. Note that $F$-axis is logarithmic (base 2 ) in these figures. When $C R=0$, i.e., no wavelength conversion is available, the effectiveness of $F$ in increasing throughput is low, unless high $F$ is assumed, in both architectures. By comparing the two figures, it is possible to see how the A-MF-SPN is able to reach high throughput even when the $C R$ is limited. For A-MF-SPN, a substantial improvement with respect to the case $C R=0$ can be obtained even with $C R=1 / 64$ for larger values of $F$. On the other hand, the AMF-SPIW requires a higher $C R$ to get high throughput; in this case improvements can be observed at $C R=1 / 16$. The maximum throughput is reached by increasing $C R$ according to the number of fibers provided; the larger the number of fibers, the lower the $C R$ needed. By combining wavelength conversion capability and multi-fiber configuration, the maximum throughput can be achieved with 
a

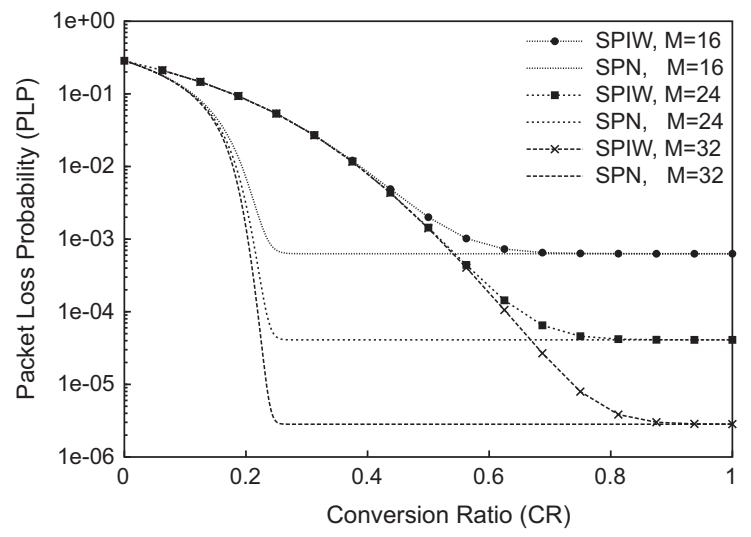

b

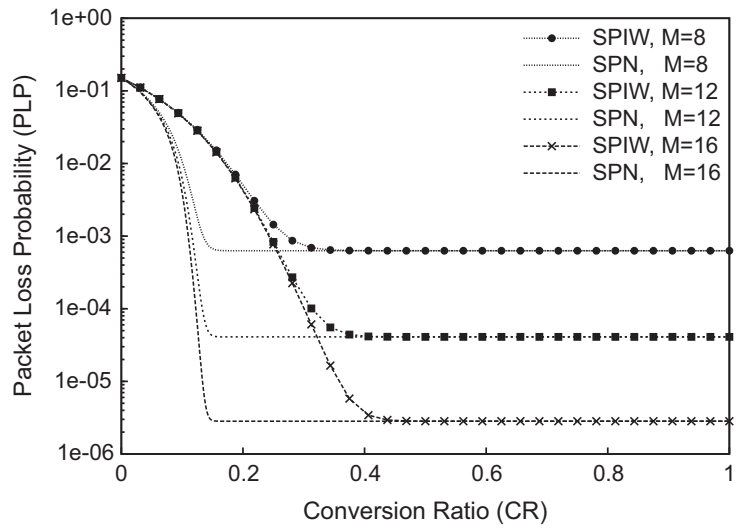

Fig. 11. Comparison between A-MF-SPN and A-MF-SPIW varying the number of channels per fiber $M$. PLP is presented as a function of the conversion ratio $C R$ as in the case $N=16, p=0.4$ and $F=1$ (a), $F=2$ (b).

reasonable values of $F$. For example, the maximum throughput can be reached in the mono-fiber case $(F=1)$ with $C R=1 / 2$ for A-MF-SPN whereas the choice of even $C R=3 / 4$ is not enough to achieve the maximum throughput for A-MF-SPIW. Having fixed the conversion ratio, the minimal number of fibers $F$ per interface can be obtained by these graphs to achieve maximum throughput. When $C R$ is high enough, the A-MF-SPIW is anyway able to provide similar throughput as the A-MF-SPN, which is important to validate that the two architectures present similar performance in this case; therefore they need to be evaluated in terms of complexity. This comparison is presented in the following section.

\subsection{Complexity comparison for A-MF-SPN and A-MF-SPIW}

This section provides a complexity comparison in terms of the number of WCs and OGs needed to reach asymptotic loss according to the switch parameters $N, M$, $F$, and p. Figs. 14(a) and (b) sketch the total number of WCs $\left(N_{W C}\right.$, axis Y1) and OGs $\left(N_{O G}\right.$, axis Y2) needed to obtain PLP asymptotic loss performance. PLP is presented as a function of $F$ ( $\log _{2} \mathrm{X}$ scale) as in the case: (a) $N=32, N_{C}=16, p=0.3$ and (b) $N=16, N_{C}=32, p=0.45$. a

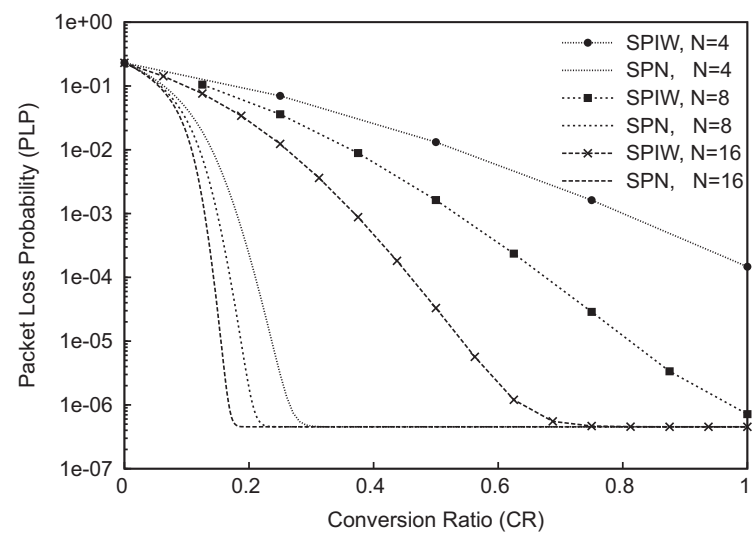

b

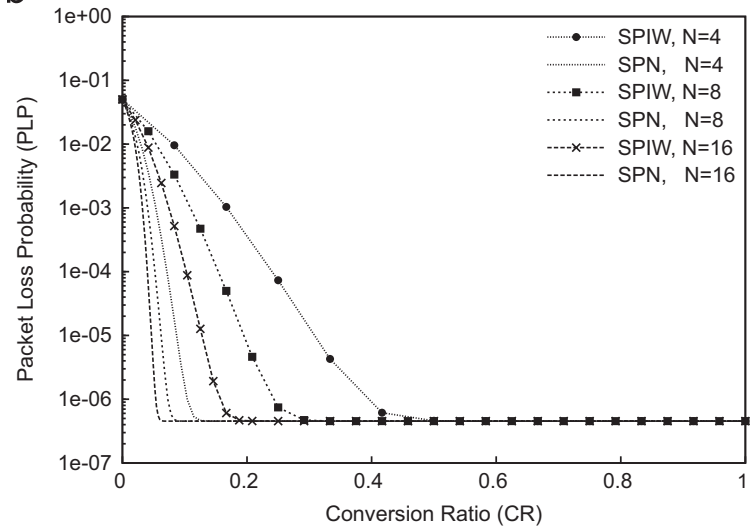

Fig. 12. Comparison between A-MF-SPN and A-MF-SPIW varying the number of II/OIs, $N$. PLP is presented as a function of the conversion ratio $C R$ as in the case $N=4,8,16, N_{C}=M F=24, p=0.3$ and $F=1$ (a), $F=3$ (b).

Under these configurations, asymptotic loss is in the range of $2 e-5$ in both cases, as shown in Fig. 10(a) and (b).

The number of WCs $N_{W C^{-}}$th needed to obtain the asymptotic PLP that are presented in Fig. 14(a) and (b), can be obtained for both A-MF-SPN and A-MF-SPIW by Fig. 10(a) and (b), respectively, recalling $C R=N_{W C} / N N_{C}$. The number of OGs $N_{O G}$ can then be obtained by formulas (1) and (2) for A-MF-SPN and A-MF-SPIW, respectively, calculated with the values $N_{W C}$-th of the previous step. Figs. 14(a) and (b) help us to draw the following key conclusions: First, the number of WCs needed, $N_{W C}$ th, rapidly decreases as $F$ increases, as expected, especially for A-MF-SPIW which requires a considerable number of WCs in the mono-fiber case. For both configurations, A-MF-SPN requires fewer WCs. When $F$ is high, the two architectures require almost the same number of WCs. The number of OGs linearly increases with $F$ for A-MFSPIW showing the same trend for both figures, while the behavior for the A-MF-SPN is strongly related to the switch configuration. In Fig. 14(a), the number of OGs required by the two architectures is not very different when $F>1$, with the A-MF-SPIW requiring a much fewer number of OGs only when $F=1$. In Fig. 14(b), instead, the A-MF-SPIW allows a relevant saving in the number of OGs 


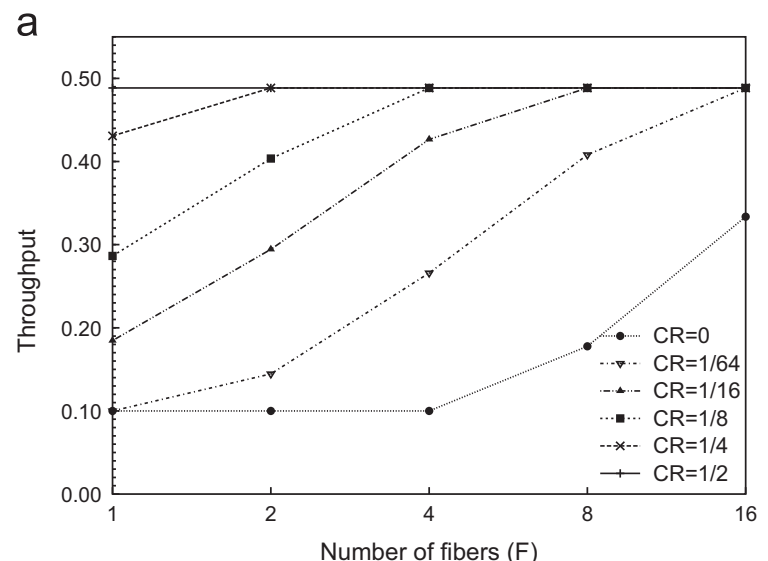

b

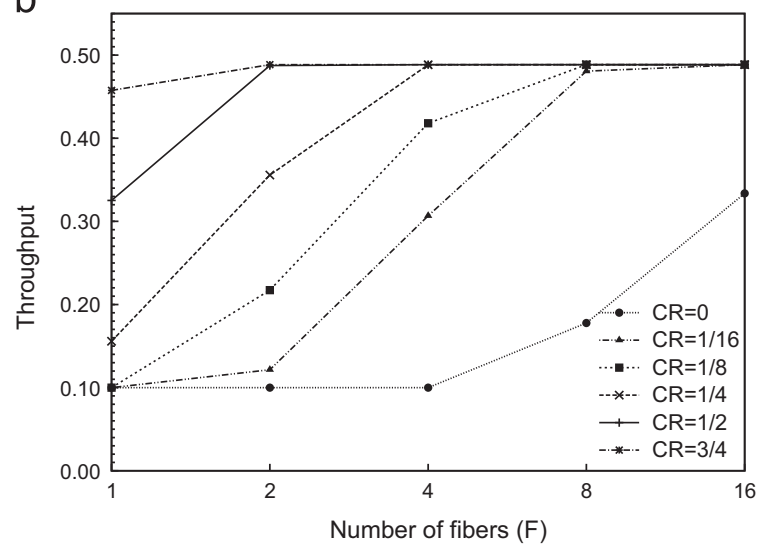

Fig. 13. Achievable throughput as a function of the number of fibers per interface $F$ varying the conversion ratio $C R$ with target $\mathrm{PLP}<1 \mathrm{e}-4$ in the case $N=16, N_{C}=32$. (a) A-MF-SPN and (b) A-MF-SPIW.

even in the multi-fiber case when $F$ is not very large, thus allowing a reduction in implementation complexity. When $F$ increases, the complexities of the two architectures in terms of optical gates tend to be the same, because in that case both formulas (1) and (2) are dominated by the first term, which is identical. It is worth to remember that the range of WCs employed in A-MFSPIW is narrower with respect to A-MF-SPN and this is expected to allow more feasible solutions.

The A-MF-SPN allows to save WCs with respect to the A-MF-SPIW, which are in the order of few hundreds or even less, in all configurations. When $N$ is small and $N_{C}$ is high (Fig. 14(b)), A-MF-SPIW scheme allows to save a relevant number of OGs, which are in the order of tens of thousands. In particular, in the cases $F=1$ and $F=2$, the A-MF-SPIW scheme allows to save a significant number of OGs, which would suggest to maintain the multi-fiber interface with a relatively small number of fibers. Hence, the A-MF-SPIW scheme saves a significant amount of OGs compared to A-MF-SPN, thus leading to potential complexity reduction, since when $F=2$, the number of WCs is also greatly reduced. Instead, when $N$ is large (Fig. 14(a)), the complexity is dominated by the number of OGs

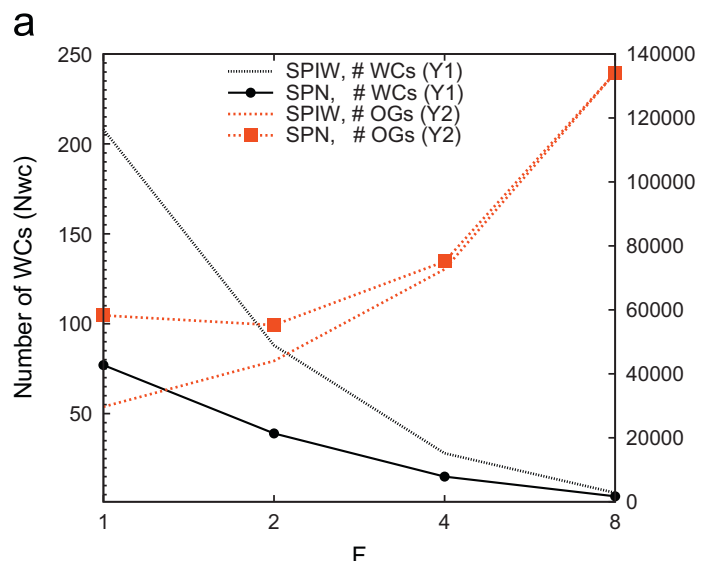

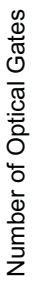

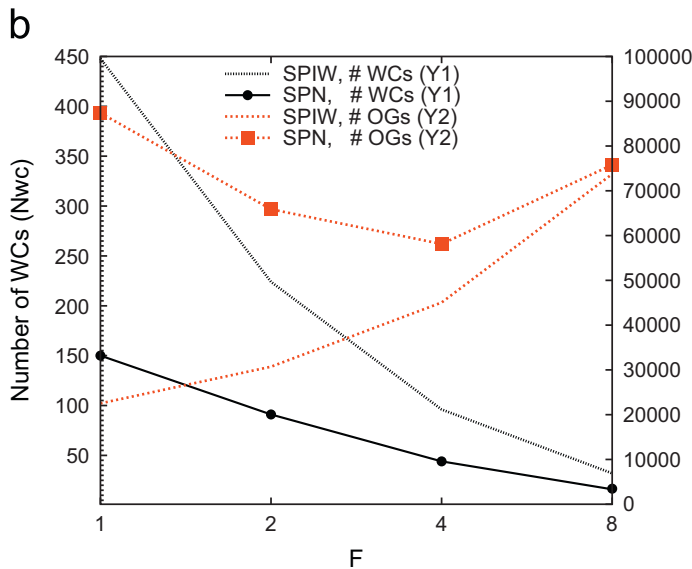

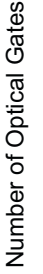

Fig. 14. Total number of WCs $N_{W C}$ (axis Y1) and OGs $N_{O G}$ (axis Y2) needed to obtain asymptotic loss (close to $2 \mathrm{e}-5$ ) for A-MF-SPN and A-MF-SPIW, as a function of $F$ as in the case: (a) $N=32, N_{C}=16, p=0.3$ and (b) $N=16, N_{C}=32, p=0.45$.

needed to interconnect the input fibers to the output fibers (first term of the formulas), so the complexity is almost the same for both sharing schemes (same number of fibers).

\section{Conclusions}

In this paper, novel state-aggregation based analytical models are presented to evaluate packet loss in asynchronous optical switches with multi-fiber interfaces and shared wavelength converters. Besides filling a methodological gap regarding A-MF-SPIW and A-MF-SPN, and validating the analytical approach against simulations, the paper produces practical numerical results and comparisons regarding the switch behavior in balanced and imbalanced traffic scenarios and also the savings in number of optical gates achieved by the A-MF-SPIW approach. The A-MF-SPIW solution is demonstrated to be a flexible approach to achieve overall switch cost optimization including both wavelength converters and space switching subsystems. 


\section{References}

[1] K. Vlachos, C. Raffaelli, S. Aleksic, N. Andriolli, D. Apostolopoulos, H. Avramopoulos, D. Erasme, D. Klonidis, M.N. Petersen, M. Scaffardi, K. Schulze, M. Spiropoulou, S. Sygletos, I. Tomkos, C. Vazquez, O. Zouraraki, F. Neri, Photonics in switching: enabling technologies and subsystem design, OSA Journal of Optical Networking 8 (May (5)) (2009) 404-428.

[2] S.J. Ben Yoo, Optical packet and burst switching technologies for the future photonic Internet, IEEE Journal of Lightwave Technology 24 (December) (2006) 4468-4492.

[3] M.J. O’Mahony, C. Politi, D. Klonidis, R. Nejabati, D. Simeonidou, Future optical networks, IEEE Journal of Lightwave Technology 24 (December (12)) (2006) 4684-4696.

[4] D. Cuda, R. Gaudino, G.A. Gavilanes, F. Neri, G. Maier, C. Raffaelli, M. Savi, Capacity/cost tradeoffs in optical switching fabrics for terabit packet switches, in: Proceedings of the ONDM 2009, Braunschweig, Germany, 18-20 February 2009.

[5] S. Yao, B. Mukherjee, S.J. Ben Yoo, S. Dixit, A unified study of contention-resolution schemes in optical packet-switched networks, IEEE Journal of Lightwave Technology 21 (2003) 672-683.

[6] Y. Fukushima, H. Harai, S. Arakawa, M. Murata, Design of wavelength-convertible edge nodes in wavelength-routed networks, Journal of Optical Networking 5 (3) (2006) 196-209.

[7] N. Akar, C. Raffaelli, M. Savi, E. Karasan, Shared-per-wavelength asynchronous optical packet switching: a comparative analysis, Computer Networks 54 (13) (2010) 2166-2181.

[8] V. Eramo, M. Listanti, P. Pacifici, A comparison study on the number of wavelength converters needed in synchronous and asynchronous all-optical switching architectures, IEEE Journal of Lightwave Technology 21 (February (2)) (2003).

[9] V. Eramo, A. Germoni, C. Raffaelli, M. Savi, Multifiber shared-perwavelength all-optical switching: architecture, control and performance, IEEE Journal of Lightwave Technology 26 (March (5)) (2008) 537-551.

[10] C. Raffaelli, M. Savi, A. Stavdas, Multistage shared-per-wavelength optical packet switch: heuristic scheduling algorithm and performance, IEEE Journal of Lightwave Technology 27 (March (5)) (2009) 538-551.
[11] C. Raffaelli, M. Savi, G. Tartarini, D. Visani, Physical path analysis in photonic switches with shared wavelength converter, in: Proceedings of the ICTON 2010, Munich, Germany, June 27-July 1, 2010.

[12] C. Raffaelli, M. Savi, Power consumption in photonic switches with shared wavelength converters, in: Proceedings of the ICTON 2010, Munich, Germany, June 27-July 1, 2010.

[13] V. Eramo, E. Miucci, A. Cianfrani, A. Germoni, M. Listanti, An analytical model evaluating the performance of small size asynchronous optical packet switches, in: Proceedings of the ICTON 2011, Stockholm, Sweden, 26-30 June 2011.

[14] N. Akar, C. Raffaelli, M. Savi, Analytical model of asynchronous shared-per-wavelength multi-fiber optical switch, in: Proceedings of the HPSR 2011, Cartagena, Spain, 4-6 July 2011.

[15] B. Mukherjee, Optical Communication Networks, McGraw-Hill, New York, 1997.

[16] L. Li, A.K. Somani, A new analytical model for multifiber WDM networks, IEEE Journal of Selected Areas in Communications 18 (October (10)) (2000) 2138-2145.

[17] A.K. Somani, M. Mina, L. Li, On trading wavelengths with fibers a cost-performance based study, IEEE/ACM Transaction on Networking 12 (October (5)) (2004) 944-951.

[18] C. Raffaelli, G. Muretto, Combining contention resolution schemes in WDM optical packet switches with multifiber interfaces, OSA Journal of Optical Networking 6 (January (1)) (2007) 74-89. Special issue on photonics in switching.

[19] V. Eramo, A. Germoni, A. Cianfrani, M. Listanti, C. Raffaelli, Evaluation of power consumption in low spatial complexity optical switching fabrics, IEEE Journal of Selected Topics in Quantum Electronics 17 (March/April (2)) (2011) 396-405.

[20] A. Stavdas, A. Bianco, A. Pattavina, C. Raffaelli, C. Matrakidis, C. Piglione, C.T. Politi, M. Savi, R. Zanzottera, Performance evaluation of large capacity broadcast-and-select optical crossconnects, Elsevier Optical switching and Networking 9 (January (1)) (2012) $13-24$.

[21] N. Akar, V. Eramo, C. Raffaelli, Comparative analysis of power consumption in asynchronous wavelength-modular optical switching fabrics, Elsevier Optical Switching and Networking 8 (July (3)) (2011) 139-148. Special issue on Green communications and networking. 